\title{
Retinopathy Based Multistage Classification of Diabeties
}

\author{
Deepak N A ${ }^{1, *}$ Savitha $\mathrm{G}^{2,}$ Deepak D J ${ }^{3}$, Supraj P. Kashyap ${ }^{4}$
}

\author{
${ }_{1,2,4}$ Computer Science and Engineering, RVITM, Bengaluru \\ ${ }^{3}$ Information Science and Engineering, RVITM, Bengaluru \\ *Corresponding author. Email: deepakna.rvitm@rvei.edu.in
}

\begin{abstract}
One of the biggest problems faced in biomedical engineering is the non-invasive assessment of the physiological changes that occur within the human body. Particularly, the detection of the abnormalities in the human eye is very difficult due to the numerous complexities involved in the process. Retinal images can be used to determine the nature of the abnormalities that affect the human eye. Standard disease identification techniques from retinal images mostly involve manual intervention. However, since human observation is extremely prone to error, the success rate of these techniques is quite scarce. Diabetic Retinopathy is one such disease of retina which occurs in people suffering from diabetes. It is a multistage progressing disease namely NDPR and PDR. Micro-aneurysms, haemorrhages and exudates are the anomalous features frequently detected in the retinal images of a person afflicted by diabetic retinopathy. Image processing techniques are applied to pre-process the Fundus image, which is followed by segmentation of anomalies. Feature extraction is done and the features that are detected are used to identify the different stages of diabetic retinopathy using Random Forest classification technique. It is observed that, the proposed algorithm results in approximate classification rate up-to $90 \%$
\end{abstract}

Keywords: Retinopathy, Feature Extraction, Retinal Image Processing, Random Forest.

\section{INTRODUCTION}

Diabetic Retinopathy (DR) is a condition of Diabetes that affects the eyes. The major cause for diabetic retinopathy is the damage to blood vessels of the retina, and the light sensitive tissues at the backside of the eye. This might not have any symptoms or may exhibit mild problems in-vision and finally, it may result in total blindness. It may even lead to blindness eventually. It amounts to one of the leading causes of blindness in the world. Around 80 percent of populations having diabetes for more than 10 years are prone to this disease. Almost 2/3 of population suffering from diabetic will be in either Type 2 or Type-1. Most of the people, who are suffering from Type-1 diabetics, are expected to develop over a period of time. The Figure 1 depicts the difference between a person with normal vision and a person affected with diabetic retinopathy. The Diabetic retinopathies are majorly categorized into two types: The 1) Non-proliferative diabetic retinopathy (NPDR) is the primitive stage of the disease in which symptoms will be existent.

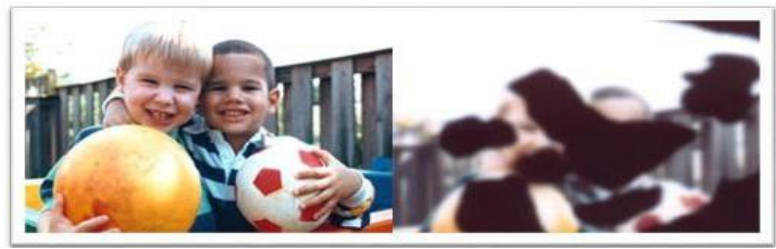

Figure 1 Vision Affected by Diabetic Retinopathy a) A person with Normal Vision b) Vision affected by Diabetic Retinopathy.

The blood vessels in the retina are weakened, in NPDR. Tiny swellings in the blood vessels, called micro aneurysms, may discharge fluid into the retina. This leakage may lead to the inflammation of the macula. 2) Proliferative diabetic retinopathy (PDR) is the next stage of the disease. Here, circulation issues cut off the supply of oxygen to the retina. Due to which new, delicate blood vessels begin to develop in the retina and into the vitreous, gel-like fluid that occupies the rear end of the eye. Hence, the new blood vessels can spill blood into the vitreous, thereby clouding the vision of a person. Additionally, issues due to the disconnection of 
the retina due to scar tissue emergence. Due to PDR, new blood vessels emerge into the eye, which removes fluid from the eye. Thereby, increasing the eye pressure, this damages the optic nerve. If this is left unaddressed, PDR can cause serious vision loss, which may finally lead to blindness. The Fundus Image shows the internal structure of the eye captured using specialist Fundus cameras which have a convoluted microscope connected to a camera that has a flash. The major compositions that can be identified on the Fundus image include the central and peripheral retina, optic disc and macula. Prevalent abnormalities constitute Micro-aneurysms, Haemorrhages and Exudates as seen in the Figure 2.

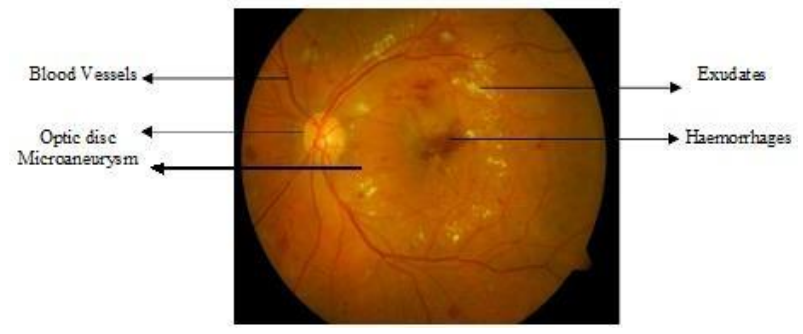

Figure 2 Fundus image containing abnormalities

\section{RELATED WORKS}

All the existing systems concentrate in segmenting the primary anomalies responsible for initial stage of the disease. The existing methods are also database dependent and would work only for that specific database. There is no single approach or method for segmenting all the anomalies. Building an optimal feature set is needed as this disease is vision threatening and high benchmarking results need to be achieved. Many classification techniques are proposed with different and novel approaches of pre-processing. Related algorithms and method are given. The different stages of Diabetic Retinopathy (DR) are identified [1]. The deformities formed in retinal blood vessels like the altered perimeter, area of spread can be used to evaluate the severity of Diabetic Retinopathy. An algorithm for identifying the different stages of diabetic retinopathy is disused [2]. The early signs of DR Micro-aneurysms are considered for classifying the different stages of the disease. Based on the number of discerned Microaneurysms, images are classified. Diabetic retinopathy patients are diagnosed based on their Fundus retinal images [3]. Their methodology uses green plane out of RGB constituent of image for increased precision. We can analyze the severity of the disease in accordance with the area of DR attribute constituent along with the aid of ANN by focusing and demarcating weights to the preceding layer. The classification of Fundus images utilizing random forest algorithm is disused [4], after the feature extraction the groups or clusters of Fundus images are formed known as classes for classification. To detect the early stage of DR, we use the features of the pre - processed image $[5,6]$, after it is pre - processed, the image is then operated morphologically using a structuring element in the form of a disk in order to extract the features. Finally, the images are classified using Support Vector Machine. All these surveyed papers achieve an average of $85 \%$ accuracy which still needs to be improved. Researches through-out the world are trying to propose better classification algorithms by building significant feature set. Limitations of the existing systems are defects that arise because of DR conformity which may not be able to be extracted utilizing single method. Thus, there is a need for a developed system in order to subdue and be able to obtain all constituents utilizing single platform.

\section{METHODS}

Proposed system is a multistage classifier of Diabetic Retinopathy. This system overcomes the drawbacks of the existing system by classifying the disease into four stages, namely, Normal, NPDR 1, NPDR 2 and PDR. This multistage classification is important because the disease itself progresses in multiple stages. The reoccurrence of the disease depends on the stage in which the treatment is provided, so it is not enough to classify the image as just normal and abnormal. The pre-processing part of both the existing and proposed system remain similar, the difference comes in the segmentation and feature extraction stages. Existing system only segmented anomalies like micro-aneurysms; the problem with this is anomaly occurs in the initial stage of the disease. Treatment cannot be given at this stage hence this is a major drawback. The proposed system overcomes this by segmenting hemorrhages along with microaneurysms and also by considering a large feature set which includes the area and count of the segmented anomalies. The feature set also includes textural features like energy and correlation, and statistical features like mean and variance. This feature set is then used to classify the image into the respective severity. The Figure 3 shows the system architecture of the proposed methodology.

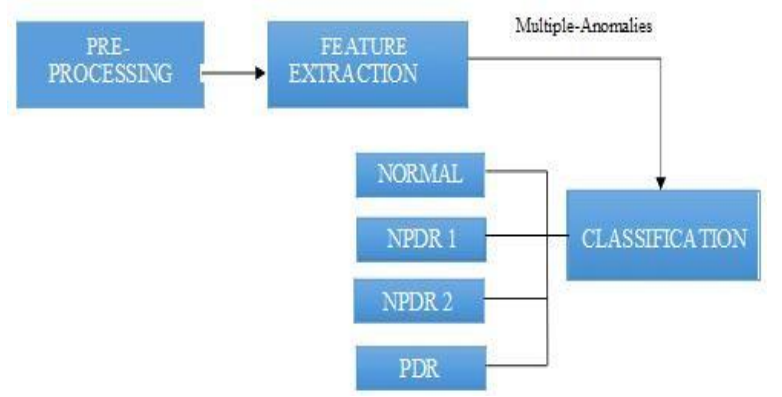

Figure 3 System Architecture - Proposed Methodology 


\subsection{Pre-processing}

The patient body motion, poor focusing of lens and improper positioning, reflection from other surfaces, bad lighting conditions will normally affect the analysis process which will lead the images to be of poor quality. In about $10 \%$ of retinal images, artifacts notably impede human grading. The pre-processing guarantees the sufficient quantity of success in the detection of abnormalities in these images. Variations can be created in retinal images because of the variations of camera positioning, lighting condition, angle in which the images are captured and retinal pigmentation.

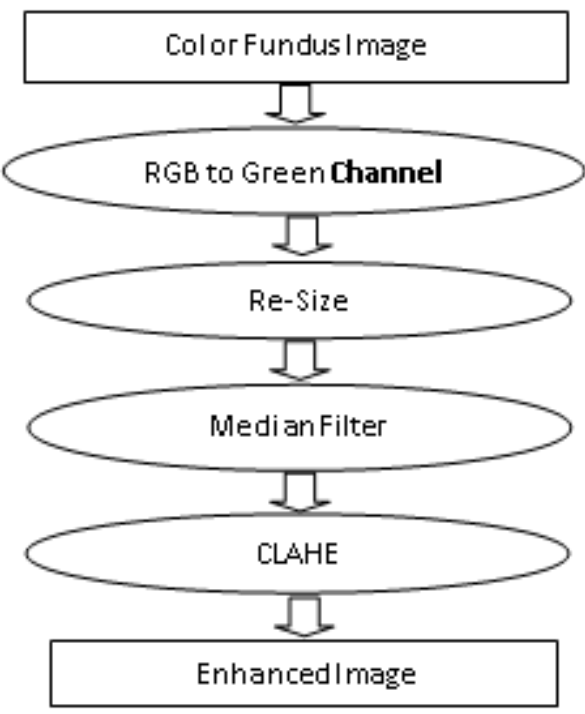

Figure 4 Steps involved in Pre-processing

Pre-processing of the images can be done, by firstly attenuating the image variations, accomplished by normalization. The normalization process is applied on the original retinal image with respect to its colour. The colour features are compared with the reference image during normalization process. The images of retina used as benchmark, often do not display high contrast. The retina images exhibit higher contrast at the center with reduction as we move outwards. Then the application of local contrast enhancement method on such images marks as a second pre-processing step of the proposed setup. The steps involved in pre-processing are shown in Figure 4.

\subsubsection{RGB to Green Channel}

The colour image is converted to a gray scale image and then the green channel is extracted from it. Green channel is better than the red or blue channels because the red channel image is too vivid and the blue channel image is too gloomy. All the anomalies are visible properly in the green channel image. A comparison of the images of the three channels is shown in Figure 5.

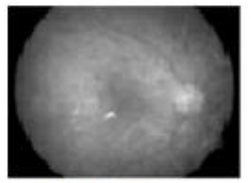

RED Channel

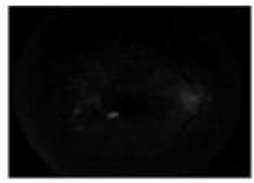

GREEN Channel

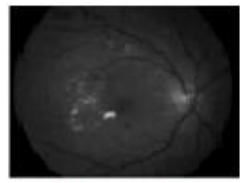

BLUE Channel
Figure 5 Red, Blue and Green Channel Images

\subsubsection{Image Resizing}

Image resize is the technique of changing the pixel information found in an image. Image resizing results in depletion in size of the image and unwanted pixel information will be discarded. In the proposed methodology, the green channel image is then resized $560 \times 720$ standard aspect ratio.

\subsubsection{Median filtering}

One of the major advantages of pre-processing an image is to remove noise. The noise from the images is removed using median filtering. The median filtering is a more efficient algorithm that removes noise effectively, while preserving the fringes of an image. In the process of noise removal, the median filter scans the image pixel by pixel and replaces each value of the pixel with the neighbouring pixels. The pixel patterns are called as "window" that slides over the entire image by scanning pixel by pixel. The median value is found from the pixels within the window. This value is used to replace the pixel being considered within the middle pixel value.

\subsubsection{CLAHE}

The CLAHE (Contrast Limited Adaptive Histogram Equalization) is one of the familiar image enhancement methodologies. In histogram equalization, the dynamic range and contrast of an image is adapted by altering the image such that its intensity histogram has a required shape. The intensity levels are re-casted such that the peaks of the histogram are stretched and the troughs are squeezed. Contrast limited adaptive histogram equalization (CLAHE) is a flexible contrast histogram equalization procedure, where the variation of an image is enhanced by applying CLAHE on small data regions called tiles rather than the entire image.

\subsection{Mask Generation-Binary Image}

The generated mask acts as binary image of same resolution, as of Fundus image. The positive pixels correspond to the fore front area. The pixels of an Fundus mask, also known as pixels within the Fundus are numbered as 1's and non-correspondent pixels of Fundus are marked as 0's Figure 6. The indigenous Fundus background is separated using RGB to HIS colour system. This involves in representing pixels in distinct channels with varying values in intensity. The 
unwanted signals from the Fundus mask are detached using median filter and erosion is done with respect to morphological processing to remove the edge pixels of the Fundus.

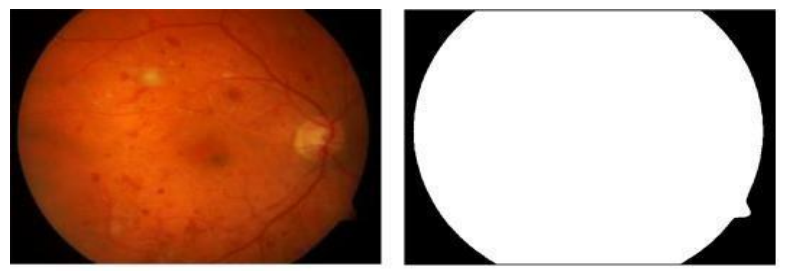

Figure 6 Input Image and the Generated mask

\subsection{Vessel detection and removal}

Blood vessel segmentation is a notable step in the red lesion detection. Since the blood vessels and red lesions namely micro- aneurysms and haemorrhages are both red in colour the blood vessels need to be pulled out of the Fundus image in order to productively recognize the micro-aneurysms and haemorrhages. The CLAHE is performed on the binary of green channel image. Top-hat filter is the analogous of subtracting the result of performing a morphological opening operation on the input image. The blood vessels are isolated using suitable threshold value; the threshold is selected based on the foregoing knowledge of the quality of the image. The resultant image is composed of blood vessels along with haemorrhages, micro-aneurisms and other stray structures. Finally, the stray structures are removed based on the area that have less than a threshold considered. The resultant image contains only blood vessels that are crucial for further processing.

\subsection{Feature Extraction}

Once the anomalies are segmented the next juncture is feature extraction. The feature which needs to be extracted is shown in Figure 7. The gray-scale image of the input image is used to extract textural features and statistical features. The textural features are obtained by using GLCM. Based on the frequency of a pixel, and its intensity value $(i)$, that occur with specific spatial relationship with the value $(j)$ is used to calculate the Grey level co-occurrence matrix. The spatial relationship of a pixel is found by considering the pixelof-interest and the pixel to its right. The other spatial relationships between the adjacent pixels can also be described. The pixel value $(i, j)$, in the GLCM of an input image, is the sum of the times the pixel with value (i), occurred in the spatial relationship to pixel value $(j)$. The co-occurrence matrix is important, that reveals several properties, such as spatial distribution of grey levels in the texture image.

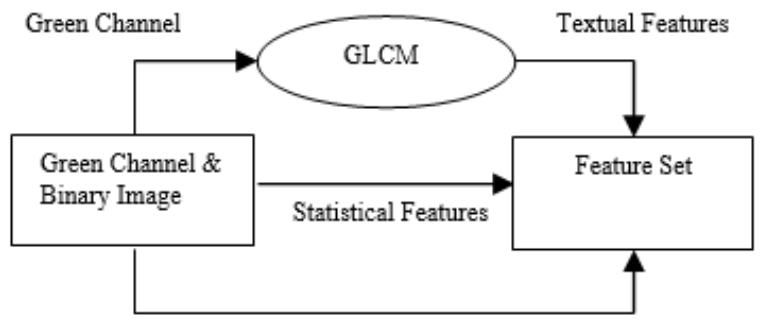

Figure 7 Feature Extractions - Textural and Statistical Features

The Figure 8, illustrates, how the extracted features are refined and gray co-occurrence matrix is generated. The element $(1,1)$ of the GLCM is assigned with value 1 , because the input image matrix contains only a single instance of adjacent pixels with the values a1 respectively. Likewise, the element $(1,2)$ is assigned with value 2 , as there are two instances. The element (1, 3 ) accommodates value 0 , because in the resultant GLCM, we cannot find any instances of it.

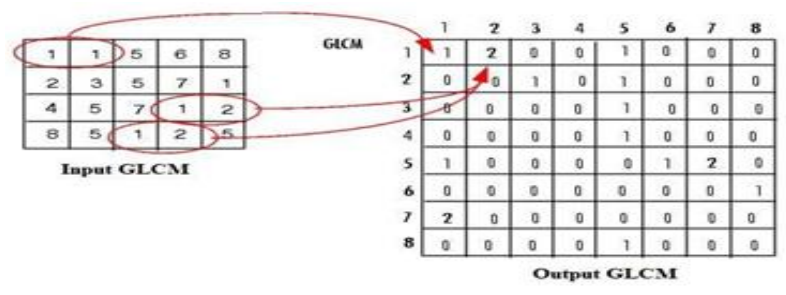

Figure 8 Construction of GLCM

This process of calculating the value is continued for all the pixels. The statistical features are calculated by applying the said technique and from the segmented anomalies the area and count is calculated. The textural features, statistical features, area and count are then fed to the classifier for further processing in the next step.

\subsection{Classification-Random Forest Algorithm}

Training statistics samples should be collected in order to establish appropriate decision rules. Categorization procedures such as supervised or unsupervised learning will then be selected on the basis of the training datasets. The proposed algorithm uses supervised machine learning algorithms that can be operated on the labelled training data, which has several training examples [13-15]. The labelled data in supervised learning algorithms contains pair of information consisting of input object and expected output value. The algorithm scrutinizes the data and fabricates the output, which is used to for plotting new instances. The impeccable results will decide the class labels for unseen instances. Depending up on the decision rule, the images are grouped into different classes. These classes represent the severity level of the disease and are represented by grades $0,1,2$ and 3, where 0 is typical, 1 is clement, 2 is average and 3 is severe. 
The Random Forests algorithm is one of the finest among classification algorithms - able to classify big chunks of data with perfection [16-21]. The random forest algorithm, also known as nearest neighbour predictor is a combo learning method used for categorization and retrogression. This classification technique erects decision trees during training. The random forest algorithm works based on the values of random vector. These random vectors are sampled unaided with the identical distribution. The idea behind random forest algorithm is that batch of weak learners form an alliance together to form a vigorous learner. They are used as instrument for making conjecture that introduces the precise kind of fortuitousness to make it as accurate classifier. The Random forest algorithm attempts to diminish the problems of high dissimilarity and high bias by a mounting to find a natural stability between the two extremes, which was major pitfall using single decision trees. Considering that Random Forests have few boundaries to calibrate and can be used simply with default boundary settings, they are a simple tool to use without having a model [22].

\section{RESULTS AND DISCUSSION}

Several experiments were accomplished to test the conduction of the suggested system. The images of Fundus dataset shown in the Figure 9 are used in the experimental process.

\subsection{Fundus Dataset}

The Fundus public dataset [7, 8] contains in-total 15 No's healthy patient images, 15 No's of images effected by diabetic retinopathy and 15 No's of images with acute glaucomatous. The image masks discovering the Field of View (FOV) are provided for specific datasets. This dataset was created by the specialists, who work in different fields of retinal image supported by ophthalmology clinics. The Figure 9 shows the images of Fundus dataset - healthy eyeball with no-infection. Similarly, the Figure 10 shows some images of Fundus dataset - with the infection in the eye ball.

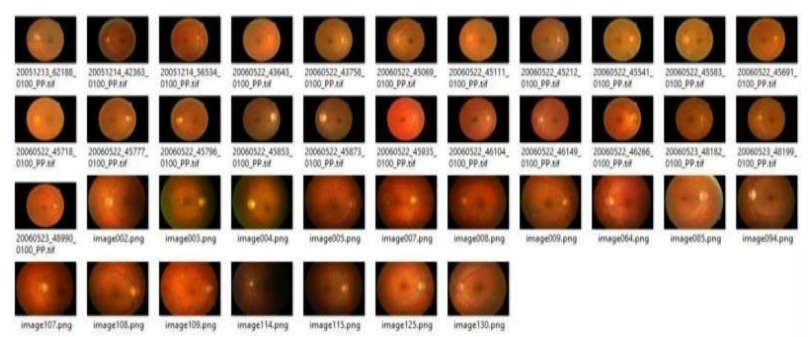

Figure 9 Fundus Dataset [9] - Fundus images of healthy eyeball

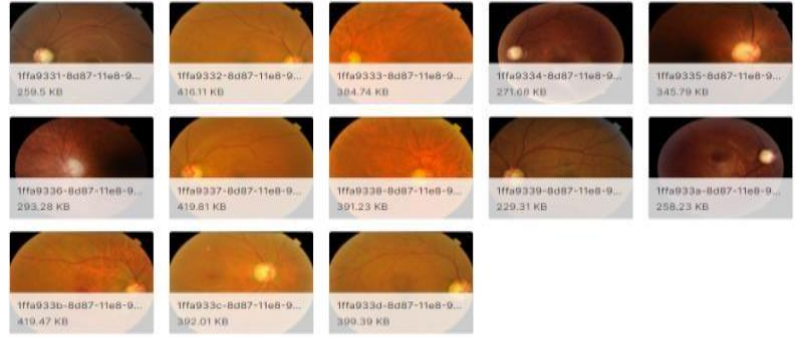

Figure 10 Fundus Dataset [9] - Fundus images of infected eye

The recognition process involves in identifying the security levels such as normal, moderately affected, highly and severely affected. This is achieved by sequence of steps like i) morphological operations that include dilation and erosion and ii) Edge detection.

\subsection{Morphological Techniques on Fundus}

They are used to acknowledge the construction or incarnation of an image. This actually means recognizing entities or restrictions within an image. The structuring element is made available to an input image using morphological operations. This also creates an output image of identical size as input image. The values of these pixels are based on the comparison of neighbouring pixels. The size and configuration of the neighbourhood is used to decide the specific morphological operation that are sensitive to specific shape and input image.

\subsubsection{Dilation and Erosion}

The Dilation and erosion are important morphological processing functions $[10,11]$.

These operations are uncomplicated set of operations and they are the basic set of elements of different algorithms. The pixels of the image, with interest produce dialation and erosion. The dilation and erosion are used to add and remove the pixels on the object boundaries [23-26]. The size and shape of the structuring element determines the number of pixels needs to attach or detached from the object. In morphological operations the state of the given pixel is decided by application of rule for the corresponding and neighbouring pixels of an input image. The Figure 11 and Figure 12 show the dilation and erosion using disk shaped structuring element.

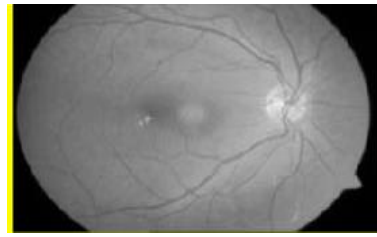

(a)

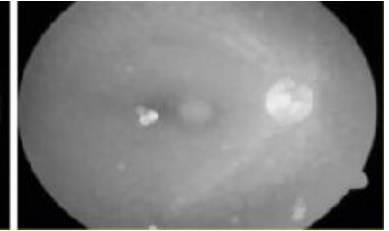

(b)
Figure 11 Dilation - a) Original Image b) Dilated Image 


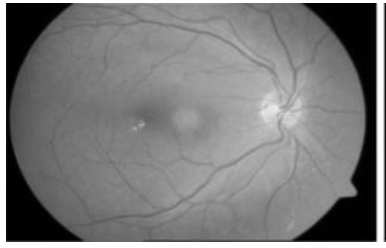

(a)

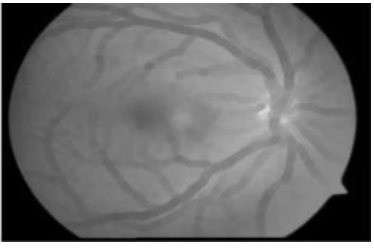

(b)
Figure 12 Erosion - a) Original Image b) Dilated Image

\subsection{Edge Detection}

In an image, an edge is something like a curve that goes behind a path of expeditious change in image intensity [12-14]. Here we use canny edge detection to recognize the edges associate with an image. To detect the strong and weak edges, the canny edge detector uses two thresholds. The weak edge will be added to output, if it is associated with a strong edge as shown in Figure 13.

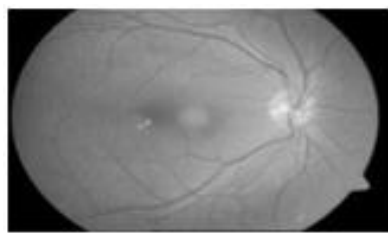

(a)

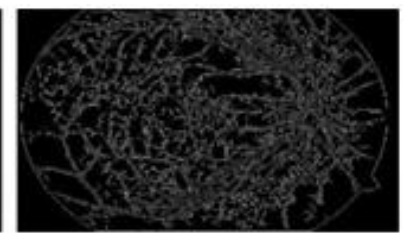

(b)
Figure 13 Erosion a) Original image; b) Edge Detected

Finally, the proposed methodology identifies the security level based on the dilation \& erosion and edges found. The obtained results are shown in the Table 1.We also collate the proposed technique with ultra-modern techniques used in finding the results.

Table1. Comparison of obtained Results

\begin{tabular}{|l|l|l|l|}
\hline Technique & Anomalies & Features Extracted & Accuracy \\
\hline Morphological processing[2] & Blood vessels & Perimeter, Area & $85 \%$ \\
\hline SVM[3] & Micro-aneurysm & Count & $83 \%$ \\
\hline ANN [4] & Micro-aneurysm exudates & Area & $86.36 \%$ \\
\hline Proposed Method & Nil & Statistical \&Textural & $90 \%$ \\
\hline
\end{tabular}

\section{CONCLUSION}

The swift and systematic early observation of Diabetic Retinopathy is only possible if there is a productive method for segregating the diabetic characteristics in the Fundus image. The preferred system presents a nimble, fruitful and sturdy way of distinguishing diabetic features in the Fundus images which can be used for classification of the images based on the seriousness of the disease. The retinal images are subjected to gray scale conversion, preprocessing and feature extraction steps. The extracted features are fed to a Random Forest classifier which will classify the images into different severity levels. Thus the Random forest technique has specified a successful DR screening method which assists to diagnose the disease in multiple phases.

\section{REFERENCES}

[1] Manjula R, Raghupathy Reddy, K. M. M. Rao, Image processing for identifying different stages of diabetic retinopathy, International Journal on Recent Trends in Engineering \& Technology, vol.11(1), 2014, pp.83-88.

[2] Soumya Sree, A. Rafega Beham, BP and SVM based diagnosis of diabetic retinopathy, International Journal of Innovative Research in Computer and Communication Engineering, vol.3(6), 2015.

[3] Mane Shreekant, Diabetic retinopathy: patient identification and measurement of the disease using ANN, International Journal of Technical Research and Applications, vol.10(31), 2015, pp. 278-282. EISSN:2320-8163.
[4] C. Aravind, Ponnibala, Vijaya chitra, Automatic detection of micro aneurysms and classification of diabetic retinopathy images using SVM technique, in Proc. of International conference on innovations in intelligent instrumentation, optimization and electrical sciences (ICIIIOES), vol.11, 2013.

[5] J. D. Labhade, L.K. Chouthmol, Diabetic retinopathy detection using random forest, International Journal of Modern Trends in Engineering and Research, 2016. EISSN: 2349-9745.

[6] Mohammed Shafeeq, B. Indira, A Survey on Automatic Detection of Diabetic Retinopathy, International Journal of Computer Engineering \& Technology (IJCET) , vol.6 (11), 2015, pp. 36-45.

[7] Jagadish Nayak, Subbanna Bhat, U. Rajendra Acharya, C. M. Lim, Manjunath Kagathi, Automated identification of diabetic retinopathy stages using digital Fundus images, International Journal of Medical Systems, vol.32(2), 2008.

[8] Sopharak, M.N.Dailey, B.Uyyanonvara, S.Barman, T. Williamson, Y.A. Moe, Machine learning approach to automatic exudates detection in retinal images from diabetic patients, Journal of modern optics, vol. 57(2), 2010, pp.124-13.

[9] G. Gary, Yen, Wen Fung Leong, Sorting system for hierarchical grading of diabetic Fundus images: A preliminary study, IEEE Transactions on Information Technology in Biomedicine, vol. 12(1), 2000, pp. 118130.

[10] L. James, Kinyoun, Donald, Martin, Y. Wilfred Fujimoto, Donna L. Leonetti, Opthalmoscopy versus Fundus photographs for detecting and Grading Diabetic Retinopathy. Morphology Fundamentals: Dilation and 
Erosion: Morphological Operations, Image Processing Toolbox ${ }^{\mathrm{TM}}$.

[11] Bernhard, Screening for diabetic retinopathy using computer based image analysis and statistical classification, Journal of Computer Methods and Programs, vol. 62, 2000, pp.165-175

[12] Wong Li Yun, U. Rajendra Acharya, Venkatesh, Caroline Chee, Lim Choo Min, K. Ng, Identification of different stages of diabetic retinopathy using retinal optical images", vol.178, 2008, pp.106-121.

[13] Arun, M., E. Baraneetharan, A. Kanchana, and S. Prabu. "Detection and monitoring of the asymptotic COVID-19 patients using IoT devices and sensors." International Journal of Pervasive Computing and Communications (2020).

[14] Seyhan, Kübra, Tu N. Nguyen, Sedat Akleylek, Korhan Cengiz, and SK Hafizul Islam. "Bi-GISIS KE: Modified key exchange protocol with reusable keys for IoT security." Journal of Information Security and Applications 58 (2021): 102788.

[15] Nguyen, Tu N., Bing-Hong Liu, Nam P. Nguyen, and Jung-Te Chou. "Cyber security of smart grid: attacks and defenses." In ICC 2020-2020 IEEE International Conference on Communications (ICC), pp. 1-6. IEEE, 2020.

[16] Pham, Dung V., Giang L. Nguyen, Tu N. Nguyen, Canh V. Pham, and Anh V. Nguyen. "Multi-topic misinformation blocking with budget constraint on online social networks." IEEE Access 8 (2020): 7887978889 .

[17] Kumar, M. Keerthi, B. D. Parameshachari, S. Prabu, and Silvia liberata Ullo. "Comparative Analysis to Identify Efficient Technique for Interfacing BCI System." In IOP Conference Series: Materials Science and Engineering, vol. 925 , no. 1, p. 012062. IOP Publishing, 2020.

[18] Rajendrakumar, Shiny, and V. K. Parvati. "Automation of irrigation system through embedded computing technology." In Proceedings of the 3rd International Conference on Cryptography, Security and Privacy, pp. 289-293. 2019.

[19] K. Yu, L. Tan, X. Shang, J. Huang, G. Srivastava and P. Chatterjee, "Efficient and Privacy-Preserving Medical Research Support Platform Against COVID-19: A Blockchain-Based Approach", IEEE Consumer Electronics Magazine, doi: 10.1109/MCE.2020.3035520.

[20] Subramani, Prabu, K. Srinivas, R. Sujatha, and B. D. Parameshachari. "Prediction of muscular paralysis disease based on hybrid feature extraction with machine learning technique for COVID-19 and post-COVID-19 patients." Personal and Ubiquitous Computing (2021): $1-14$.

[21] Z. Guo, Y. Shen, A. K. Bashir, M. Imran, N. Kumar, D. Zhang and $\mathrm{K}$. $\mathrm{Yu}$, "Robust Spammer Detection Using Collaborative Neural Network in Internet of Thing Applications", IEEE Internet of Things Journal, vol. 8, no. 12, pp. 9549-9558, 15 June15, 2021, doi: 10.1109/JIOT.2020.3003802.
[22] L. Tan, H. Xiao, K. Yu, M. Aloqaily, Y. Jararweh, "A Blockchain-empowered Crowdsourcing System for 5Genabled Smart Cities", Computer Standards \& Interfaces, https://doi.org/10.1016/j.csi.2021.103517.

[23] C. Feng et al., "Efficient and Secure Data Sharing for 5G Flying Drones: A Blockchain-Enabled Approach," IEEE Network, vol. 35, no. 1, pp. 130-137, January/February 2021, doi: 10.1109/MNET.011.2000223.

[24] N. Shi, L. Tan, W. Li, X. Qi, K. Yu, "A BlockchainEmpowered AAA Scheme in the Large-Scale HetNet", Digital Communications and Networks, https://doi.org/10.1016/j.dcan.2020.10.002.

[25] Y. Sun, J. Liu, K. Yu, M. Alazab, K. Lin, "PMRSS: Privacy-preserving Medical Record Searching Scheme for Intelligent Diagnosis in IoT Healthcare", IEEE Transactions on Industrial Informatics, doi: 10.1109/TII.2021.3070544.

[26] Kumar, M. Keerthi, B. D. Parameshachari, S. Prabu, and Silvia liberata Ullo. "Comparative Analysis to Identify Efficient Technique for Interfacing BCI System." In IOP Conference Series: Materials Science and Engineering, vol. 925, no. 1, p. 012062. IOP Publishing, 2020. 